\title{
Interview with Alexander Cohen
}

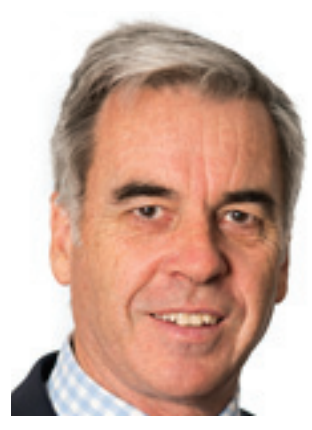

Ander Cohen speaks to Adam Price-Evans, Commissioning Editor of Future Cardiology: Alexander (Ander) Cohen MBBS (Hons), MSc, MD, FRACP is a vascular physician and epidemiologist at Guy's and St Thomas' Hospital, King's College (London, UK). He graduated with honors in medicine and honors in surgery from the University of Melbourne, Australia, and became a fellow of the Royal Australasian College of Physicians in 1990. He was awarded an MSc in Epidemiology from the London School of Hygiene and Tropical Medicine, University of London in 1991 with a thesis on the metabolic syndrome in South-Asian populations. In 1998, he was awarded an MD with a thesis on the epidemiology of venous thromboembolism and thromboprophylaxis. In addition to his clinical work, he is involved in designing, managing and analyzing clinical trials from Phase I to IV. $\mathrm{He}$ is the Chairman and a member of many international steering committees for multicenter trials, epidemiological and pharmacoeconomic studies, and was previously the Director of Clinical Research and an Epidemiologist in Thrombosis Research at King's College Hospital.

First draft submitted: 22 March 2017; Accepted for publication: 27 March 2017; Published online: 1 June 2017

\section{Q One of your primary focuses is} research into venous thromboembolism. Could you tell us a bit more about venous thromboembolism \& the current treatment guidelines?

Venous thromboembolism (VTE) is a relatively common condition. In fact, it is the third most common cause of cardiovascular death after stroke and myocardial infarction. It is often underdiagnosed because the symptoms and signs are not specific. The current treatment guidelines are anticoagulation for 3-6 months or even longer for patients with certain types of venous thrombosis, such as when the thrombosis is unprovoked or related to ongoing or permanent risk factors. The anticoagulation treatment favored by the treatment guidelines are direct oral anticoagulant (DOAC) agents because they seem to be just as effective as vitamin $\mathrm{K}$ antagonists (VKAs) such as warfarin, however, are clearly much safer.

Q As you say, the field is now looking
toward DOACs for treatment of recurrent
VTE instead of aspirin or warfarin. Why
do you think that such alternative
methods have been called for \& what are
the proposed benefits associated with
using DOACs?
First, the DOACS are simpler to use. They
do not require routine monitoring and
they offer oral therapies that allow easier
outpatient treatment and shorter duration
of hospital stay. This has been seen consist-
ently with the DOAC therapies. Second,

\section{KEYWORDS}

- aspirin • direct oral anticoagulants

- EINSTEIN-CHOICE • rivaroxaban

- venous thromboembolism 
I think they are called for because not only are they as effective as vitamin $\mathrm{K}$ antagonists, which help prevent recurrent VTE, when used in this setting they are also safer. This is clear when you consider that bleeding into the brain and death from bleeding are both reduced by two-thirds when compared with VKAs. Also, major bleeding is reduced by approximately $50 \%$ and clinically relevant nonmajor bleeds are reduced by at least $25 \%$. Together, the evidence shows that DOACs are more tolerable, have a better safety profile and are more convenient to use than other alternatives such as VKAs.

Q You are a principal investigator for the Phase III EINSTEIN-CHOICE trial, which involves the study of long-term rivaroxaban treatment of VTE. Could you please tell me a bit more about the study \& its primary goals \& objectives?

The EINSTEIN-CHOICE trial was a study of patients that have already received a therapeutic course of 6-12 months anticoagulation for the treatment of their VTE and there was equipoise regarding the need for extended anticoagulation. It was a randomized double-blind multicenter trial where patients were split into three treatment arms and received either once-daily rivaroxaban $20 \mathrm{mg}$, rivaroxaban $10 \mathrm{mg}$ or aspirin $100 \mathrm{mg}$. The primary goal of the study was to demonstrate that rivaroxaban $20 \mathrm{mg}$ or rivaroxaban $10 \mathrm{mg}$ once-daily was superior to aspirin for preventing recurrent VTE.

Q Recently, results from the trial were presented at the 2017 American College of Cardiology session in Washington DC. Could you please describe the results presented?

The results showed that rivaroxaban $20 \mathrm{mg}$ and rivaroxaban $10 \mathrm{mg}$ were both superior to aspirin in preventing recurrent VTE and they both showed no significant increase in major bleeding when compared with aspirin.

Q What do you think are the main implications of these results \& how do you think they will impact clinical practice for VTE in the future? Would you say we have sufficient evidence to recommend rivaroxaban over cheaper alternatives? I think the main implication of these results is that there now appears to be no role for aspirin in preventing VTE. We now have a low dose of rivaroxaban (10 mg once-daily) which is significantly more effective than aspirin and has similar safety. I believe aspirin had a role up until the completion of this study as it was demonstrated to result in a modest $30 \%$ reduction in VTE compared with placebo; however, anticoagulants had been shown to reduce thrombosis by approximately $80-90 \%$. We have therefore now demonstrated with this randomized double-blind study that there are no advantages of aspirin over anticoagulants and, in fact, there are only disadvantages with respect to reducing thrombosis. Another major implication of this study is that we now know that both doses of rivaroxaban (20 and $10 \mathrm{mg}$ ) appear to have a good safety profile and are highly effective. This provides physicians with a choice when prescribing rivaroxaban and allows them to adjust the dose depending on how they assess the risk of recurrence in the patient. So together, I think we now have sufficient evidence to say that there are benefits of rivaroxaban over and above cheaper alternatives such as low-dose aspirin.

Q Do you think there are any further barriers to overcome before physicians will routinely prescribe rivaroxaban for recurrent VTE? Yes, I think there are still a number of barriers to overcome. There is a challenge related to current practices as many physicians prescribe anticoagulants for 3 or 6 months and do not always consider the risk of recurrence. This may be problematic because patients who have an unprovoked or what used to be known as an idiopathic VTE have high recurrence rates and will require longer periods of therapy, particularly when treating a recurrent VTE episode, in which case therapy may be continued indefinitely. However, patients who have a clear major predisposing or provoked VTE, such as a thrombosis provoked by major surgery or major trauma, generally only need 3 months of treatment. There is however an entire intermediate group of patients that we have looked at in this study that have relatively minor provoking risk factors or persistent provoking factors such as history of cancer or thrombophilia. These patients might actually require longer term therapy. In the EINSTEIN-CHOICE study, we showed that these intermediate patients with minor provoked VTE or persisting risk factors have a significant burden of recurrence if they are treated with aspirin; however, this can be reduced by treating with rivaroxaban instead. I therefore believe we have a major educational 
challenge, not only in relaying the overall findings, which show that the rivaroxaban was more efficacious than the aspirin, but also in how to recognize whether a patient should be provided with extended therapy. These barriers will therefore require ongoing education and discussion.

Q What do you think will or should be the next stages in VTE research?

I think in this particular area of research, we now have strong evidence showing that in patients with persistent risk factors, treatment should continue for longer than 3 months and as shown in EINSTEIN-CHOICE and other extended treatment studies for at least another year and there is no reason to believe that risk of recurrence will diminish after that stage. We now also need to perform longer term studies to evaluate patients who have been receiving treatment for several years and to assess what happens when therapy is stopped. This of course cannot always be performed in clinical trials and therefore pharmacoepidemiological studies will likely be required. Additionally, while we now know that the lower $10-\mathrm{mg}$ dose of rivaroxaban is an effective treatment option in this area, however we are currently unaware as to whether even lower doses would be effective, which is another possible area of study. We also need to collect more information on minor provoked VTE patients with persistent risk factors in order to build risk factor profiles. This will allow us to assess whether certain groups of patients are at a particularly low or high risk of recurrence. There are therefore a number of avenues for further study and I am sure research will continue in these areas.

\section{Disclaimer}

The opinions expressed in this interview are those of the interviewee and do not necessarily reflect the views of Future Medicine Ltd.

Financial \& competing interests disclosure

$A$ Cohen receives consulting fees from Aspen, Bayer, Boehringer-Ingelheim, Bristol-Myers Squibb, CSL Behring, Daiichi-Sankyo, EKOS, GlaxoSmithKline, GLG, Guidepoint Global, Johnson and Johnson, Leo Pharma, McKinsey, JP Morgan, Navigant, ONO, Pfizer, Portola, Sanofi, Takeda, Temasek Capital, TRN, XO1; has an advisory board membership with Bayer, Bristol-Myers Squibb, Daiichi-Sankyo, Johnson and Johnson, ONO, Pfizer, Portola, Sanofi, XO1; receives payments for lectures including speakers bureau services, payments for preparation of reports and payment for development of educational presentations from, Aspen, Bayer, Boehringer-Ingelheim, Bristol-Myers Squibb, Daiichi, GlaxoSmithKline, Johnson and Johnson, Medscape, Pfizer and Portola. He is an advisor to the UK Government Health Select Committee, the all-party working group on thrombosis, the Department of Health and the NHS, on the prevention of VTE. He is an advisor to the charity "Lifeblood: the thrombosis charity" and is the founder of the European educational charity, "The Coalition to Prevent Venous Thromboembolism". The author has no other relevant affiliations or financial involvement with any organization or entity with a financial interest in or financial conflict with the subject matter or materials discussed in the manuscript apart from those disclosed.

No writing assistance was utilized in the production of this manuscript. 